\title{
Temporary left ventricular assist device may be safer than veno-arterial extracorporeal membrane oxygenation for treating shock in the presence of a mitral prosthesis
}

\author{
Yuting P. Chiang, MD, ${ }^{\mathrm{a}}$ Alina Nicoara, MD, ${ }^{\mathrm{b}}$ and Carmelo A. Milano, MD, ${ }^{\mathrm{a}}$ Durham, NC
}

From the ${ }^{\mathrm{a} D i v i s i o n}$ of Cardiothoracic Surgery, Department of Surgery, and ${ }^{\mathrm{b}}$ Department of Anesthesiology, Duke University Medical Center, Durham, NC.

Disclosures: Dr Milano discloses a PILVAS LV vent system patent. All other authors reported no conflicts of interest.

The Journal policy requires editors and reviewers to disclose conflicts of interest and to decline handling or reviewing manuscripts for which they may have a conflict of interest. The editors and reviewers of this article have no conflicts of interest.

Received for publication April 13, 2020; revisions received April 13, 2020; accepted for publication April 30, 2020; available ahead of print May 11, 2020.

Address for reprints: Yuting P. Chiang, MD, Division of Cardiothoracic Surgery, Department of Surgery, Duke University Medical Center, Box 3043, Durham, NC 27710 (E-mail: Ypc2@ duke.edu).

JTCVS Techniques 2020;3:206-8

2666-2507

Copyright (C) 2020 The Authors. Published by Elsevier Inc. on behalf of The American Association for Thoracic Surgery. This is an open access article under the CC BY-NC-ND license (http://creativecommons.org/licenses/bync-nd/4.0/).

https://doi.org/10.1016/j.xjtc.2020.04.020

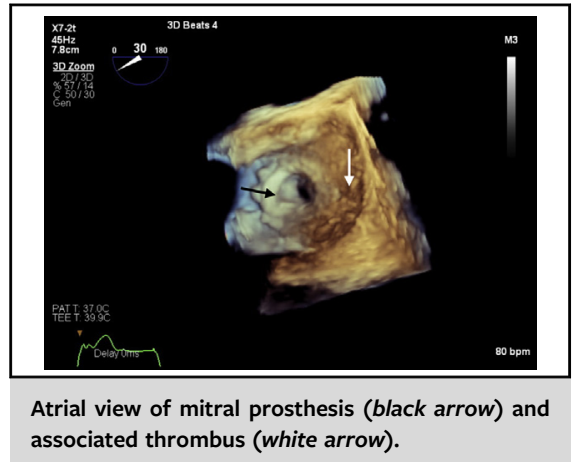

CENTRAL MESSAGE

Temporary left ventricular assist device with apical inflow may be safer than veno-arterial membrane oxygenation for supporting patients in cardiogenic shock who had undergone recent mitral valve replacement.

See Commentaries on pages 209 and 211.
Intracardiac thrombosis (ICT) is a devastating complication of veno-arterial extracorporeal membrane oxygenation (VA ECMO). ${ }^{1,2}$ Reduced intracardiac flow due to decreased preload and increased afterload is part of the presumed mechanism. A recently implanted mitral prosthesis may exacerbate this situation. We describe mitral prosthesis thrombosis in a patient supported with VA ECMO who was successfully bridged to recovery using a temporary left ventricular assist device (LVAD) with apical inflow to maintain flow across the prosthesis. This study was approved by the Duke Health Institutional Review Board; the requirement for informed consent was waived.

\section{CASE DESCRIPTION}

A 40-year old female who had undergone previous mitral valve repair (26-mm ring with closure of P1-P2 and P2-P3 clefts) presented to an outside hospital with dizziness and orthopnea in the setting of moderate-to-severe mitral stenosis (mean gradient, $17 \mathrm{~mm} \mathrm{Hg}$; mean valve area, $1.3 \mathrm{~cm}^{2}$ ) but normal left ventricular (LV) function. Coronary angiography revealed nonobstructive disease. Elective redo mitral valve replacement was performed.

Video clip is available online. 


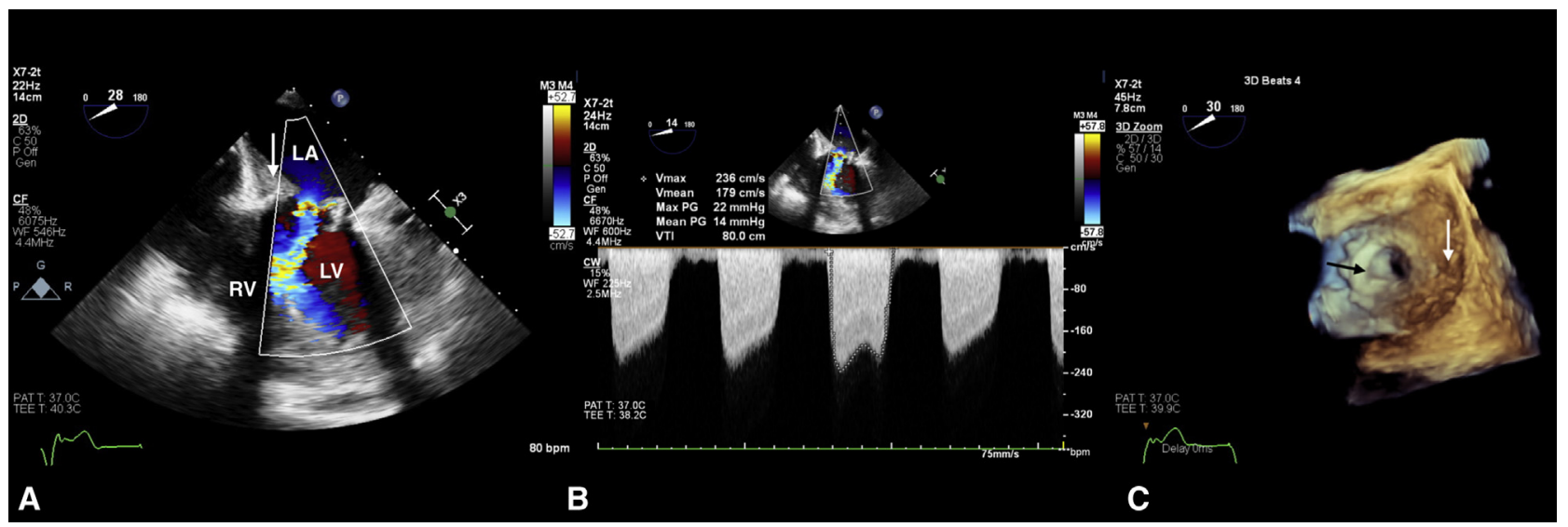

FIGURE 1. Thrombus partially occluding a recently implanted mitral prosthesis that developed during veno-arterial extracorporeal membrane oxygenation support. (A) Thrombus (white arrow) layering the left atrial wall. Color flow Doppler reveals accelerated blood flow due to partial occlusion of the prosthesis. (B) Elevated mean gradient (14 mm Hg) across the mitral orifice due to partial occlusion of the prosthesis. (C) Three-dimensional en-face atrial view of prosthesis (black arrow) and thrombus (white arrow). $L A$, Left atrium; $R V$, right ventricle; $L V$, left ventricle; $P G$, pressure gradient; $V T I$, velocity time integral.

marginal with retrograde filling of the circumflex. On POD 3 , repeat TEE demonstrated persistently depressed LV function and a thrombus on the mitral prosthesis. The patient was then transferred to our institution.

On POD 4, the patient underwent reoperation for left atrial thrombus removal and temporary LVAD implantation. Intraoperative TEE showed a thrombus partially occluding the prosthesis (Figure 1 and Video 1). After thrombus removal, the prosthesis displayed a reduced gradient and no insufficiency (Figure 2 and Video 1). The ascending aorta and LV apex were cannulated with $19 \mathrm{Fr}$ and $36 \mathrm{Fr}$ cannulae, respectively, and connected to a Rotaflow LVAD. Her LV demonstrated recovery over the next 2 weeks.

On POD 10, an intra-aortic balloon pump was placed, and the temporary LVAD was weaned. CPB was instituted using the existing aortic cannula and a femoral venous cannula, facilitating removal of the apical cannula and ventriculotomy repair. The patient was weaned off $\mathrm{CPB}$ and returned to the intensive care unit in stable condition. The intra-aortic balloon pump was removed on POD 15. Subsequent echocardiography demonstrated improved LV function (ejection fraction $30 \%$ ), free movement of all 3 mitral prosthetic leaflets, a mean gradient of 8 to $10 \mathrm{~mm} \mathrm{Hg}$, and no regurgitation. The patient's course was subsequently prolonged by renal failure and infections, but no further cardiovascular interventions were required, and the patient was discharged on POD 97.

\section{DISCUSSION}

The combination of a recently implanted mitral prosthesis and VA ECMO may increase the risk of ICT. ${ }^{3}$ Diminished preload and increased afterload resulting from VA ECMO support can result in altered or absent flow across the prosthesis, which is more prone to thrombosis in the early postoperative period. There has been 1 previous case report describing ICT in the setting of VA ECMO and a recently implanted mitral bioprosthetic. ${ }^{4}$

Although surgical vents (placed via the pulmonary vein or LV apex) can reduce LV distention, flow across the prosthesis would still be limited primarily by the preload reaching the left heart, which is significantly reduced on VA ECMO. Percutaneous devices such as the Impella (Abiomed, Danvers, Mass) face the same limitation. A temporary LVAD with apical cannulation is a more robust approach to guarantee flow across the prosthesis and can be considered from the start as an alternative to VA ECMO. This approach maintains the entire cardiac output

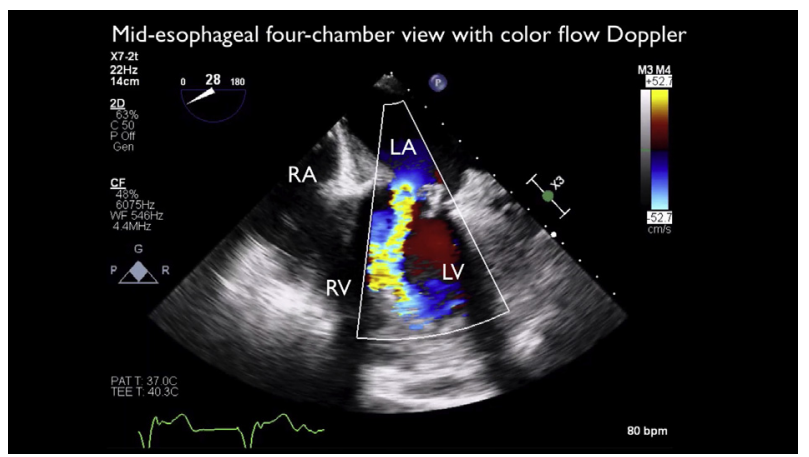

VIDEO 1. (A) Intraoperative preprocedure transesophageal echocardiography (TEE) examination performed on veno-arterial extracorporeal membrane oxygenation (VA ECMO). (B) Intraoperative postprocedure TEE examination performed after removal of thrombus, placement of a temporary left ventricular assist device, and VA ECMO decannulation. $R A$, Right atrium; $L A$, left atrium; $R V$, right ventricle; $L V$, left ventricle; $M V$, mitral valve. Video available at: https://www.jtcvs.org/article/S2666-2507(20) 30211-X/fulltext. 


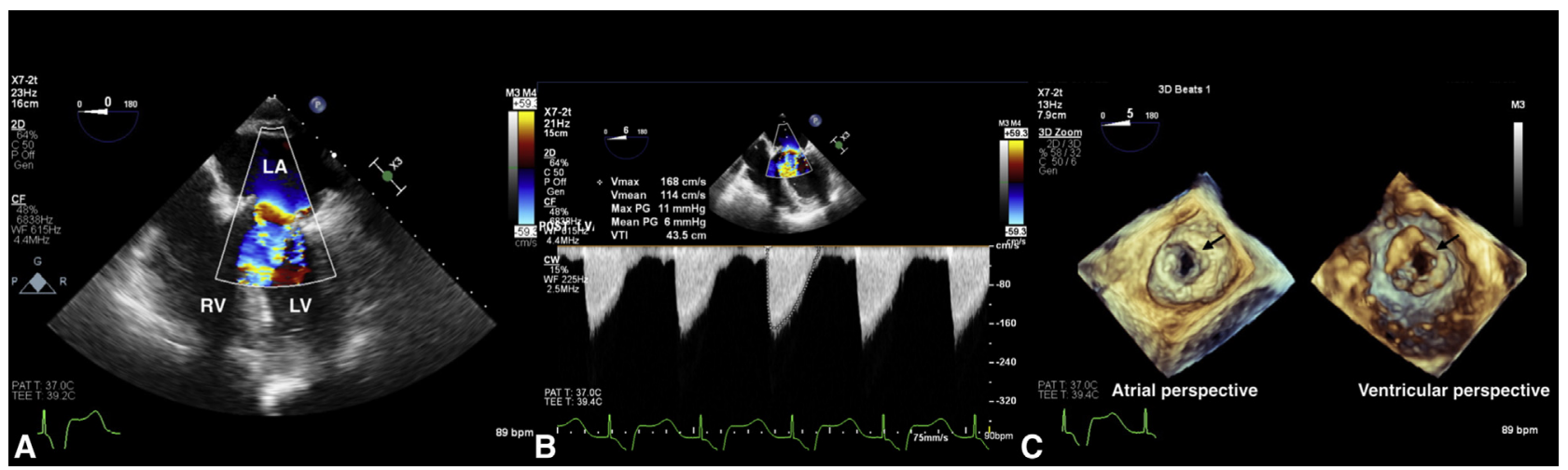

FIGURE 2. Improved flow across mitral prosthesis after thrombus evacuation and conversion from veno-arterial extracorporeal membrane oxygenation to a temporary extracorporeal left ventricular assist device with apical cannulation. (A) Improved flow across the mitral prosthesis demonstrated by color flow Doppler. (B) Improved mean gradient (6 mm Hg) across the prosthesis after thrombus evacuation. (C) Three-dimensional en-face views of prosthesis (black arrow) after thrombus evacuation. $L A$, Left atrium; $R V$, right ventricle; $L V$, left ventricle; PG, pressure gradient; $V T I$, velocity time integral.

across the prosthesis. Disadvantages include increased technical difficulty during implantation due to LV apical cannulation and the need for cardiopulmonary bypass during decannulation; performing a ventriculotomy on stunned or ischemic myocardium may also increase the risk.

In summary, a recently implanted mitral prosthesis may increase the risk of ICT in patients requiring VA ECMO support. Extracorporeal LVAD support with apical cannulation may prevent thrombus formation through greater blood washing of the prosthesis.

\section{References}

1. Alhussein M, Moayedi Y, Posada JD, Ross H, Hickey E, Rao V, et al. Ventricular thrombosis post-venoarterial extracorporeal membrane oxygenation. Circ Heart Fail. 2017;10:e003757.

2. Weber C, Deppe AC, Sabashnikov A, Slottosch Y, Kuhn E, Eghbalzadeh K, et al. Left ventricular thrombus formation in patients undergoing femoral veno-arterial extracorporeal membrane oxygenation. Perfusion. 2018;33:283-8.

3. Williams B, Bernstein W. Review of venoarterial extracorporeal membrane oxygenation and development of intracardiac thrombosis in adult cardiothoracic patients. J Extra Corpor Technol. 2016;48:162-7.

4. Gottfried R, Paluszkiewicz L, Kizner L, Morshuis M, Koertke H, Gummert J. Thrombosis of a bioprosthetic mitral valve under extracorporeal membrane oxygenation: thrombus formation in the left heart. Ann Thorac Surg. 2012;94:657. 